Macmillan Law Masters

Law of the European Union 


\section{Macmillan Law Masters}

\section{Law Series Editor Marise Cremona}

Basic English Law (2nd edn) W. T. Major

Business Law Stephen Judge

Company Law (2nd edn) Janet Dine

Constitutional and Administrative Law (2nd edn) John Alder

Contract Law (2nd edn) Ewan McKendrick

Conveyancing (2nd edn) Priscilla Sarton

Criminal Law Marise Cremona

Employment Law (2nd edn) Deborah J. Lockton

Family Law Kate Standley

Land Law (2nd edn) Kate Green

Landlord and Tenant Law (2nd edn) Margaret Wilkie and Godfrey Cole

Law of the European Union (2nd edn) Jo Shaw

Law of Succession Catherine Rendell

Law of Trusts Patrick McLoughlin and Catherine Rendell

Legal Method (2nd edn) Ian McLeod

Torts Alastair Mullis and Ken Oliphant 


\title{
Law of the European Union
}

\author{
Second Edition
}

\section{Jo Shaw}

Professor of European Law, University of Leeds

Law series editor: Marise Cremona

Senior Fellow, Centre for Commercial Law Studies

Queen Mary and Westfield College, University of London 
C) Jo Shaw 1993, 1996

All rights reserved. No reproduction, copy or transmission of this publication may be made without written permission.

No paragraph of this publication may be reproduced, copied or transmitted save with written permission or in accordance with the provisions of the Copyright, Designs and Patents Act 1988, or under the terms of any licence permitting limited copying issued by the Copyright Licensing Agency, 90 Tottenham Court Road, London W1P 9HE.

Any person who does any unauthorised act in relation to this publication may be liable to criminal prosecution and civil claims for damages.

First published 1993 as European Community Law

Reprinted three times

Second edition published 1996 by

MACMILLAN PRESS LTD

Houndmills, Basingstoke, Hampshire RG21 6XS

and London.

Companies and representatives

throughout the world

ISBN 978-0-333-66481-0 ISBN 978-1-349-14127-2 (eBook)

DOI 10.1007/978-1-349-14127-2

A catalogue record for this book is available from the British Library.

$\begin{array}{rrrrrrrrrr}10 & 9 & 8 & 7 & 6 & 5 & 4 & 3 & 2 & 1 \\ 05 & 04 & 03 & 02 & 01 & 00 & 99 & 98 & 97 & 96\end{array}$

Copy-edited and typeset by Povey-Edmondson

Okehampton and Rochdale, England 


\section{Contents}

Preface and Acknowledgements

xiii

Table of Cases

xiv

Table of Treaties Establishing the European Communities and the European Union

Table of UK Statutes

xliv

List of Abbreviations

xlv

\section{Part I INTRODUCING THE EUROPEAN UNION}

1 Studying the law of the European Union 3

1.1 Beginning the law of the European Union 3

1.2 The European Union and the European Community: the basic facts

1.3 The mission of the European Union 9

1.4 Key terms 11

1.5 An overview of some key elements of the legal order of the EU 15

1.6 Legal literature 20

1.7 Making the most of studying EC law 23

Summary 24

Questions $\quad 24$

Further reading $\quad 24$

2 Evolving from Community to Union 26

2.1 Introduction 26

2.2 The roots of European integration 27

2.3 The postwar climate of change 28

2.4 From grand ideals to incremental stages 30

2.5 The non-participants in supranational Europe 33

2.6 The early years 34

2.7 De Gaulle and the Luxembourg accords 35

2.8 The years of stagnation 37

2.9 Widening and deepening 39

2.10 The relaunch of the Community 40 
2.11 The Single European Act 42

2.12 After the Single European Act 44

2.13 The social dimension of the internal market 46

2.14 Towards a Treaty on European Union 47

2.15 After the IGCs: the struggle for ratification of
'Maastricht'

2.16 The Treaty of Maastricht 50

2.17 Internal policy after the end of 1992

2.18 Progress towards Economic and Monetary Union 54

2.19 The 'Third Pillar', the Schengen agreements, and immigration and asylum policies $\quad 55$

2.20 External trade and the conclusion of the Uruguay round

2.21 The development of the Common Foreign and Security policy

2.22 Responding to changed political and economic circumstances in Europe

2.23 Towards the 1996 IGC 57

Summary $\quad 59$

Questions $\quad 60$

$\begin{array}{ll}\text { Further reading } & 60\end{array}$

3 Constitutional Fundamentals of European Union 62

3.1 Introduction $\quad 62$

3.2 A constitution for the European Union? 63

3.3 The German challenge to European constitutionalism

3.4 Constitution-building in the European Union 66

3.5 A democratic system? 68

3.6 Union of peoples or union of people? Citizenship

3.7 A federal system? 76

$\begin{array}{lll}3.8 & \text { Exclusive and shared competence } & 79\end{array}$

3.9 Limited powers and implied powers $\quad 80$

3.10 Application of the principles of subsidiarity and
proportionality

3.11 A just system? $\quad 86$

3.12 A polity in a global system?

3.13 A single coherent system of law? 94

3.14 An irreversible system?

3.15 An economic constitution? 100

3.16 Conclusion 101

Summary 102

Questions $\quad 102$

Workshop 103

Further reading 103 


\section{Part II THE EUROPEAN UNION AT WORK}

4 The Institutions of the European Union

4.1 Introduction

4.2 The European Commission: composition and basic character

4.3 The policy-making function

4.4 The executive and administrative function

4.5 The representative function

4.6 Guardian of the treaties

4.7 The Council of the European Union: composition and basic character

4.8 The Council acting as an intergovernmental body

4.9 The European Council

4.10 The Committee of Permanent Representatives (COREPER)

4.11 The Presidency

4.12 The European Parliament: composition, basic character and powers

4.13 The Economic and Social Committee (ECOSOC) and the Committee of the Regions

4.14 Agencies and other bodies established under the EU Treaties

4.15 The Court of Auditors

4.16 The European Investment Bank (EIB)

4.17 The institutions of Economic and Monetary Union

4.18 The Court of Justice and the Court of First Instance Summary

Questions

Workshop

Further reading

5 The European Union and its Institutions at Work

5.1 Introduction

5.2 The Legal Acts of the institutions

5.3 Introducing the law-making process

5.4 The 'old' procedure

5.5 The cooperation procedure

5.6 Council-Parliament co-decision

5.7 The assent procedure

5.8 Common Foreign and Security Policy (CFSP) and Cooperation in Justice and Home Affairs (CJHA)

5.9 Law-making and the policy-making process reviewed

5.10 Legislation post-Maastricht: the 'simplification' programme

5.11 Comitology and the delegation of powers 
5.12 The finances of the European Union 162

5.13 The budgetary process 166

5.14 The range of Community powers 168

5.15 Interinstitutional relationships in the EU 171

5.16 Interinstitutional agreements 172

5.17 Litigation between the institutions 173

Summary 176

$\begin{array}{ll}\text { Questions } & 177\end{array}$

Further reading 178

6 Sources of Law in the European Union 179

6.1 Introduction 179

6.2 The founding treaties 179

6.3 Other international agreements 180

6.4 General principles of law 180

6.5 Principles of administrative and legislative legality 183

6.6 The pillars of economic integration as general principles of EC law $\quad 187$

6.7 Fundamental rights 188

6.8 The scope of EU fundamental rights protection 191

6.9 Fundamental rights outside the European Court 195

6.10 Political rights 197

6.11 Acts of the institutions - EU legislative and 198

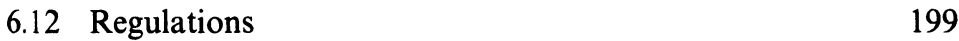

6.13 Directives 200

6.14 Decisions 201

6.15 Sui generis acts 201

6.16 'Soft law' 202

6.17 The case law of the Court of Justice and the Court of

First Instance 203

Summary 204

Questions 205

Further reading 205

7 The Implementation and Enforcement of EC Law 207

$\begin{array}{ll}7.1 & \text { Introduction } \\ 7 & 207\end{array}$

7.2 The role of Article $5 \mathrm{EC}$ in the implementation and
enforcement of EC law

7.3 Enforcement proceedings under Articles 169-171 EC 210

7.4 The range of national conduct capable of engaging state responsibility $\quad 212$

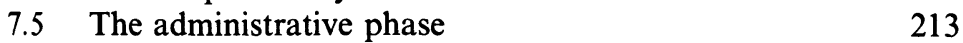

7.6 The judicial phase 216

7.7 Questions of national law in general 216 
7.8 Legislative paralysis and change of government

7.9 Defences related to the nature of the relevant provision of EC law

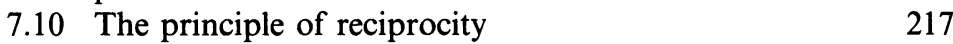

7.11 Expedited proceedings 218

7.12 Enforcement and the internal market 218

7.13 Enforcement and Economic and Monetary Union 220

7.14 Interim measures 220

$\begin{array}{ll}7.15 & \text { Sanctions } \\ 7.16 & 221\end{array}$

7.16 The individual and the Commission's enforcement powers 222

7.17 The problem of non-compliance 224 Summary $\quad 226$

Questions 227

Workshop 227

Further reading 228

Part III THE EU LEGAL ORDER AND THE NATIONAL LEGAL ORDERS

8 Article 177 EC - the Organic Connection between National

Courts and the Court of Justice 231

8.1 Introduction

8.2 Court controls on the preliminary reference procedure

8.3 Provisions of EC law which may be the subject of a reference

8.4 Courts and tribunals of the Member States capable of making a reference

8.5 The discretion to refer: Article 177(2) 239

8.6 The obligation to refer: Article 177 (3) 241

8.7 Rulings on validity 244

8.8 The authority and effects of rulings of the Court of Justice

8.9 The assessment of the preliminary reference procedure

Summary

Questions

Workshops

Further reading

9 EC Law and the Legal Systems of the Member States

9.1 The nature of the EU legal order and its impact upon the national legal orders 
9.2 The penetration of EC law into the national legal orders

9.3 EC law as a superior source of law

9.4 National constitutions and the reception of EC law

9.5 Conceptions of international law in the Member States

9.6 EC law and the sovereignty of Parliament

9.7 The instrumentalisation of the constitutional qualities of EC law

9.8 The justiciability of EC law in national courts

9.9 Prerequisites of direct effect

9.10 Provisions of EC law capable of judicial enforcement 265

9.11 Horizontal and vertical direct effect 267

9.12 The notion of an 'emanation of the state'

9.13 The effect of EC law on national law and national legislative competence

9.14 The development of the interpretative obligation

9.15 The scope of the indirect effect principle

9.16 The relationship between direct effect and indirect effect

9.17 The responsibility of the State in respect of breaches of EC law

9.18 The clarification of Francovich I

Summary

Questions

Workshop

Further reading

10 The National Dimension: Domestic Remedies for Breach of EC Law and National Reactions to the Challenge

10.2 National remedies and breach of EC law 286

10.3 National remedies and the disapplication of national law

10.4 Adequate sanctions and EC law

10.5 Effective remedies and the procedural and jurisdictional conditions applying to national remedies

10.6 The conditions governing the principle of State liability

10.7 The alternative: legislative action 294

10.8 Domestic responses to the challenges of EC law 295 Summary 299

Questions $\quad 300$

Further reading $\quad 300$ 


\section{Part IV THE JUDICIAL CONTROL OF THE EU INSTITUTIONS}

11 Introduction to the Judicial Control of the EU Institutions

11.1 Introduction

11.2 The framework of Treaty provisions

304

11.3 The many functions of the system of judicial protection

11.4 Articles 173 and 175 and public interest litigation

11.5 The division of jurisdiction and the appellate role of the Court of Justice

Summary

Questions

Workshop

Further reading

12 Judicial Review of the Acts of the Institutions

12.1 Introduction

12.2 The notion of a reviewable act

12.3 Locus standi

12.4 Locus standi: non-privileged applicants

12.5 Direct concern

12.6 Individual concern

12.7 Challenges to regulations

12.8 Can individuals challenge directives?

12.9 Quasi-judicial determinations

12.10 Locus standi of non-privileged applicants: conclusions and critique

12.11 Time limits 334

12.12 Interim measures 334

12.13 Grounds for review 335

12.14 The consequences of annulment 337

12.15 Indirect challenge 337

12.16 Article 184: the plea of illegality 338

12.17 The scope of indirect challenge under Article $177 \quad 339$

Summary $\quad 340$

Questions 341

Further reading 341

13 Judicial Control of Failure to Act 343

13.1 Introduction: Article 175 and Article 173

13.2 Procedure 344

13.3 Failure to act: privileged applicants 346

13.4 Failure to act: non-privileged applicants 347

Summary $\quad 349$

Questions $\quad 349$

Further reading 349 
14 Non-contractual Liability and Compensation for Loss Caused by the EU

14.1 Introduction 350

14.2 The conditions of liability 350

14.3 The requirement of a wrongful act 351

14.4 Wrongful acts: vicarious liability and the
responsibility of the institutions

14.5 Wrongful acts: carelessness within the Commission 352

14.6 Acts or omissions? 353

14.7 Wrongful acts: acts having legal effects 354

14.8 The relationship between annulment and compensation

14.9 The elaboration and application of the Schöppenstedt formula

14.10 Liability in respect of other acts having legal effects 360

14.11 The requirements of causation and damage 361

14.12 The problem of concurrent liability: national court or Court of Justice? 362

Summary 364

Questions $\quad 365$

Workshop 365

Further reading 366

\section{Part V CONCLUSION}

15 The Constitutional and Institutional Foundations of the European Union: Review and Conclusions

$\begin{array}{lll}15.1 & \text { Introduction } & 369\end{array}$

15.2 Law and European integration 370

15.3 The scope of the economic and social law of the EU 373

15.4 The institutional and constitutional foundations of the EU reviewed $\quad 375$

$\begin{array}{ll}\text { Bibliography } & 378\end{array}$

Index 391 


\section{Preface and Acknowledgements}

In response to the ever increasing volume of material on EC law, it was decided for this second edition to create two separate books on the law of the constitution and institutions of the EU, and on the social and economic law of the EU. As this book shows, it is not only the Court of Justice (and the Court of First Instance) which have been active in a law-making capacity since 1993 and the publication of the first edition. In addition, there are substantial bodies of institutional practice which have changed in order to absorb and respond to the novelties of the Treaty of Maastricht, and the institutions continue to be active in the legislative and 'soft-law-making' spheres. It is hoped that the approach taken here not only reflects the current 'new institutionalist' mantra that 'institutions matter' but also puts across the idea that 'institutions are interesting'. The book will have succeeded in its purpose if it continues to be used - like the first edition - across a range of disciplines and in countries with sharply differing legal systems and conceptions of the EU legal order.

This book is a greatly expanded and adjusted version of Chapters 1-10 of European Community Law (1993). New chapters have been added on EU constitutionalism, and by way of a conclusion. The material on the relationship between EC law and national law, and on the control of the EU institutions has been substantially expanded and updated and divided into additional chapters. As before, I have attempted to write an introductory text, which stimulates the reader and leads onto additional reading (of cases and of secondary literature), rather than to make a definitive statement of the law. The approach is in general terms 'contextual', and is influenced by the development of a larger body of work which considers EC law and EU institutions from an interdisciplinary perspective.

A number of colleagues and friends have continued to be a source of support. They are too numerous to mention individually, but I would like to thank in particular Kenneth Armstrong, Gráinne de Búrca, Tammy Hervey and Gillian More. I would like to thank Jo Hunt for diligent proofreading. As ever I owe a great debt to Sally Wheeler, never one of nature's Europhiles, but nonetheless consistently supportive of my endeavours. However, she was not on hand when Leo Shaw's school was closed by flooding after Easter. Leo showed remarkable (but not perfect) patience as I struggled with producing the final manuscript. I would also like to thank my parents for their practical assistance and support. Finally I would like to dedicate this book to the memory of Brian Wilkinson, a good friend and sadly missed. 


\section{Table of Cases}

\section{A: Cases before the European Court of Justice}

\section{By Case Number (in year order)}

Case 8/55 Fédéchar $v$. High Authority [1956] ECR 292

Case 9/56 Meroni $v$. High Authority [1957-58] ECR 133

Cases 1, 14/57 Société des Usines à Tubes de la Sarre $v$. High Authority [1957] ECR 105

Case 3/59 Germany v. High Authority [1960] ECR 53

Case 6/60 Humblet $v$. Belgium [1960] ECR $559 \quad 278$

Case 13/61 Bosch $v$. de Geus [1962] ECR 45

Cases 16 and 17/62 Confédération Nationale des Producteurs de

Fruits et Légumes $v$. Council [1962] ECR 471

Case 24/62 Commission v. Germany (Brennwein) [1963] ECR 63

Case 25/62 Plaumann v. Commission [1963] ECR $95 \quad 323,325,354$

Case 26/62 Van Gend en Loos $v$. Nederlandse Administratie der

Belastingen [1963] ECR 1

$34,247,252,262-3,283$

Cases 28-30/62 Da Costa en Schaake NV [1963] ECR 31 239, 242, 245

Cases 31, 33/62 Wöhrmann v. Commission [1962] ECR 501

Case 15/63 Lassalle v. Parliament [1964] ECR 31

Cases 90 and 91/63 Commission $v$. Luxembourg and Belgium

(Dairy Products) [1964] ECR 625

218

Cases 106-107/63 Toepfer $v$. Commission [1965] ECR $405 \quad 323,360$

Case 6/64 Costa v. ENEL [1964] ECR $585 \quad 34,233,252,257$, $261,270,283,338$

Case 21/64 Dalmas $v$. High Authority [1965] ECR 175

Case 16/65 Firma Schwarze $v$. Einfuhr- und Vorratstelle für

Getreide und Futtermittel [1965] ECR $877 \quad 142,233$

Case 32/65 Italy $v$. Commission [1966] ECR $389 \quad 339$

Case 48/65 Lütticke $v$. Commission [1966] ECR $19 \quad 215,348$

Case 57/65 Lütticke $v$. HZA Saarlouis [1966] ECR 205

Case 61/65 Vaassen [1966] ECR $261 \quad 238$

Cases 5, etc./66 Kampffmeyer v. Commission [1967] ECR $245 \quad 360$

Cases 8-11/66 Cimenteries v. Commission (Noordwijks Cement Accoord) [1967] ECR 75

Case 28/67 Molkerei Zentrale Westfalen $v$. HZA Paderborn [1968] ECR 143

Cases 10, 18/68 Eridania v. Commission [1969] ECR 459 
Case 29/68 Milch- Fett- und Eierkontor v. HZA Saarbrücken [1969] ECR 165

Case 4/69 Lütticke v. Commission [1971] ECR 325

Case 9/69 Sayag $v$. Leduc [1969] ECR 329

Cases 19, etc./69 Richez-Parise v. Commission [1970] ECR $325 \quad 353$

Case 29/69 Stauder $v$. City of Ulm [1969] ECR 419

Case 31/69 Commission $v$. Italy (Export Rebates) [1970] ECR $25 \quad 214$

Case 69/69 Alcan v. Commission [1970] ECR 385 321

Case 77/69 Commission v. Belgium (Pressed Wood) [1970] ECR 237

Case 9/70 Grad [1970] ECR 825

Case 11/70 Internationale Handelsgesellschaft [1970] ECR 1125

Case 15/70 Chevalley $v$. Commission [1970] ECR 975

Case 22/70 Commission v. Council (ERTA) [1971] ECR 263

Case 25/70 Einfuhr- und Vorratstelle $v$. Köster [1970] ECR $1161 \quad 160$

Cases 41-44/70 International Fruit Company v. Commission [1971] ECR 411

Case 59/70 Netherlands $v$. Commission [1971] ECR 639

Case 62/70 Bock $v$. Commission [1971] ECR 897

Case 78/70 Deutsche Grammophon v. Metro-SB-Grossmärkte [1971] ECR 487

Case 5/71 Zuckerfabrik Schöppenstedt $v$. Council [1971] ECR $975 \quad 356$

Case $7 / 71$ Commission $v$. France (Euratom Supply Agency) [1971] ECR 1003

Cases 9, 11/71 Compagnie d'Approvisionnement $v$. Commission [1972] ECR 391

Case 42/71 Nordgetreide $v$. Commission [1972] ECR 105

Case 43/71 Politi $v$. Italian Minister of Finance [1971] ECR 1039

Case 48/71 Commission $v$. Italy (Art Treasures II) [1972] ECR 527

Case 96/71 Haegemann v. Commission [1972] ECR 1005

Cases 2-4/72 International Fruit Company NV v. Produktschap voor Groenten en Fruit [1972] ECR 1219

Case 4/73 Nold $v$. Commission [1974] ECR 503

Case 8/73 Hauptzollamt Bremerhaven v. Massey-Ferguson [1973] ECR 897

Case 34/73 Variola $v$. Italian Finance Administration [1973] ECR 981

Case 166/73 Rheinmühlen-Düsseldorf $v$. Einfuhr- und

Vorratstelle für Getreide und Futtermittel [1974] ECR 33 232, 239

Case 167/73 Commission $v$. France (French Merchant Seamen) [1974] ECR 359)

Case 9/74 Casagrande $v$. Landeshauptstadt München [1974] ECR 773

Case 17/74 Transocean Marine Paint Association v. Commission [1974] ECR 1063 
Case 26/74 Roquette $v$. Commission [1976] ECR 677

Case 41/74 Van Duyn $v$. Home Office [1974] ECR 1337

Case 74/74 CNTA v. Commission [1975] ECR 533

Case 23/75 Rey Soda v. Cassa Conguaglio Zucchero [1975]

ECR 1279

Case 36/75 Rutili $v$. Minister for the Interior [1975] ECR 1219

Case 43/75 Defrenne $v$. SABENA [1976] ECR 455

Case 60/75 Russo v. AIMA [1976] ECR 45

Cases 3/76 etc. Kramer [1976] ECR 1279

Case 26/76 Metro-SB-Großmärkte v. Commission [1977] ECR 1875

Case 33/76 Rewe-Zentralfinanz $v$. Landwirtschaftskammer für das Saarland [1976] ECR 1989

Case 41/76 Donckerwolcke $v$. Procureur de la République [1976] ECR 1921

Case 45/76 Comet $v$. Produktschap [1976] ECR 2043

Cases 64, etc./76 Dumortier et al. v. Council and Commission (Quellmehl and Gritz) [1979] ECR 3091

Cases 83, etc./76 Bayerische HNL et al. v. Council and Commission (Second Skimmed Milk Powder case) [1978] ECR 1209

Case 101/76 KSH v. Commission [1977] ECR 797

Case 107/76 Hoffmann-La-Roche $v$. Centrafarm [1977] ECR 957

Case 114/76 Bela Mühle Josef Bergman v. Grows-Farm (Skimmed Milk Powder) [1977] ECR 1211

Cases 117, etc./76 Ruckdeschel v. HZA Hamburg St-Annen [1977] ECR 1753

Case 30/77 R v. Bouchereau [1977] ECR 1999

Cases 31 and 53/77R Commission v. United Kingdom (Pig Producers) [1977] ECR 921

Case 61/77R Commission $v$. Ireland (Fisheries) [1977] ECR 937

Cases 103, 145/77 Royal Scholten-Honig (Holdings) Ltd $v$.

Intervention Board for Agricultural Produce [1978]

ECR 2037

Case 106/77 Amministrazione delle Finanze dello Stato v. Simmenthal SpA (Simmenthal II) [1978] ECR 629

Case 113/77 NTN Toyo Bearing Co. v. Council and Commission [1979] ECR 1185

Case 113/77R NTN Toyo Bearing Co. v. Commission [1977]

ECR 1721

Cases 116, 124/77 Amylum and Tunnel Refineries v. Council and Commission [1979] ECR 3479

Case 143/77 KSH NV v. Council and Commission [1979] ECR 3583 
Cases 103-9/78 Beauport v. Council and Commission [1979] ECR 17

Case 120/78 Rewe-Zentrale AG v. Bundesmonopolverwaltung für Branntwein (Cassis de Dijon) [1979] ECR 649

Case 125/78 GEMA $v$. Commission [1979] ECR 3173

Case 141/78 France $v$. United Kingdom [1979] ECR 2923

Case 148/78 Ratti [1979] ECR 1629

Case 232/78 Commission v. France (Lamb Wars) [1979]

ECR 2729

Case 4/79 Providence Agricole de la Champagne [1980] ECR $2823 \quad 246$

Case 44/79 Hauer $v$. Land Rheinland Pfalz [1979] ECR 3740

Case 61/79 Denkavit Italiana [1980] ECR 1205

Case 104/79 Foglia $v$. Novello (No. 1) [1980] ECR 745

Case 131/79 R $v$. Secretary of State for Home Affairs, ex parte Santillo [1980] ECR 1585

Case 138/79 Roquette Frères $v$. Council [1980] ECR 3333

Case 155/79 A.M. \& S. v. Commission [1982] ECR 1575

Case 730/79 Philip Morris Holland BV v. Commission [1980] ECR 2671

Cases 789-790/79 Calpak SpA v. Commission [1980] ECR 1949

Case 811/79 Amministrazione delle Finanze dello Stato $v$. Ariete SpA [1980] ECR 2545

Cases 36, 71/80 Irish Creamery Milk Suppliers Association $v$. Ireland [1981] ECR 735

Case 66/80 International Chemical Corporation $v$.

Amministrazione delle Finanze dello Stato [1981]

ECR 1191

$242,245-6$

Case 158/80 Rewe-Handelsgesellschaft Nord mbH $v$.

Hauptzollamt Kiel [1981] ECR 1805

287

Case 203/80 Casati [1981] ECR 2595

Case 244/80 Foglia $v$. Novello (No. 2) [1981] ECR 3045

Case 246/80 Broeckmeulen [1981] ECR 2311

Case 269/80 R v. Tymen [1981] ECR 3079

273

Case 8/81 Becker [1982] ECR 53

Case 60/81 IBM v. Commission [1981] ECR 2639

Case 96/81 Commission $v$. Netherlands [1982] ECR 1791)

Case 102/81 Nordsee $v$. Reederei Mond [1982] ECR 1095

Case 104/81 Kupferberg [1982] ECR 3659

Case 108/81 Amylum v. Council [1982] ECR 3107

Case 136/81 Commission v. Italy [1982] ECR 3547

Case 211/81 Commission v. Denmark [1982] ECR 4547

Case 230/81 Luxembourg v. Parliament [1983] ECR 255 
Case 283/81 CILFIT [1982] ECR 3415

Case 307/81 Alusuisse v. Council and Commission [1982] ECR 3463

Cases $314 / 81$, etc. Procureur de la République $v$. Waterkeyn [1982] ECR 4337

Case 11/82 Piraiki-Patraiki v. Commission [1985] ECR 207

Case 74/82 Commission $v$. Ireland (Imports of poultry) [1984]

\section{ECR 317}

Case 162/82 Cousin [1983] ECR 1101

Case 191/82 FEDIOL $v$. Commission [1983] ECR 2913

Case 199/82 Amministrazione delle Finanze dello Stato v. San Giorgio [1983] ECR 3595

Cases 205-15/82 Deutsche Milchkontor GmbH v. Germany [1983] ECR 2633

Case 216/82 Universität Hamburg v. HZA Hamburg-Kehrwieder [1983] ECR 2771

Cases 228, 229/82 Ford of Europe Inc $v$. Commission [1984] ECR 1129

Case 231/82 Spijker Kwasten v. Commission [1983] ECR 2559

Cases 239, 275/82 Allied Corporation v. Commission [1984] ECR 1005

Case 264/82 Timex $v$. Council and Commission [1985] ECR $849 \quad 332$

Case 322/82 Commission $v$. France [1983] ECR 3705

Case 13/83 Parliament $v$. Council (Transport Policy) [1985] ECR 1513
$119,173,311,345-6$

Case 14/83 Von Colson and Kamann $v$. Land Nordrhein

Westfalen [1984] ECR $1891 \quad 209,267,272-4,289$

Case 16/83 Prantl [1984] ECR 1299

271

Case 145/83 Adams $v$. Commission [1985] ECR 3539

352,362

Case 222/83 Municipality of Differdange $v$. Commission [1984] ECR 2889

Case 240/83 Procureur de la République $v$. Association de défense des brûleurs d'huiles usagées [1985] ECR 520

182

Case 288/83 Commission $v$. Ireland (Potatoes) [1985] ECR 1761

Case 293/83 Gravier $v$. City of Liège [1985] ECR 593

215

Case 294/83 Parti Ecologiste 'Les Verts' v. European Parliament [1986] ECR 1339 $16,87,174,202,303,315-16,320,326$, $337,339,348$

Case 1/84R Ilford $v$. Commission [1984] ECR 423 334

Case 41/84 Pinna $v$. Caisse d'allocations familiales de la Savoie [1986] ECR 1

Case 44/84 Hurd $v$. Jones [1986] ECR 29

Cases 60-1/84 Cinéthèque $v$. Fédération nationale des cinémas français [1985] ECR 2605

Cases 142 and 156/84 BAT and Reynolds $v$. Commission (Philip Morris) [1987] ECR 4487 
Case 152/84 Marshall $v$. Southampton and South West

Hampshire AHA (Marshall I) [1986] ECR 723

$267-9,297$

Case 169/84 COFAZ v. Commission [1986] ECR 391

Case 175/84 Krohn v. Commission [1986] ECR 753

Case 178/84 Commission v. Germany [1987] ECR 1227

Case 181/84 R v. Intervention Board for Agricultural Produce, ex parte Man (Sugar) Ltd [1985] ECR 2889

Case 222/84 Johnston $v$. Chief Constable of the Royal Ulster

Constabulary [1986] ECR $1651 \quad 86,188,267,270,274,276,286,329$

Case 253/84 GAEC v. Council and Commission [1987] ECR $123 \quad 361$

Case 25/85 Nuovo Campsider v. Commission [1986] ECR $1531 \quad 344$

Case 48/85 Commission v. Germany [1986] ECR 2549

Case 85/85 Commission v. Belgium [1986] ECR 1149

Case 97/85 Union Deutsche Lebensmittelwerke v. Commission [1987] ECR 2265

Case 133-6/85 Walter Rau v. BALM [1987] ECR 2289

Case 281, etc./85 Germany et al. v. Commission (Migration Policy) [1987] ECR 3203

Case 293/85 Commission $v$. Belgium [1988] ECR 305

Case 314/85 Firma Foto-Frost v. Hauptzollamt Lübeck [1987] ECR 4199

Case 1/86 Commission v. Belgium (Water Pollution) [1987] ECR 2797

Case 12/86 Demirel $v$. Stadt Schwäbisch Gmund [1987] ECR 3719

Case 14/86 Pretore di Salò v. X. [1987] ECR 2545

Case 34/86 Council v. Parliament [1986] ECR 2155

167,316

Case 45/86 Commission $v$. Council (Generalised Tariff Preferences) [1987] ECR 1493

Case 60/86 Commission $v$. UK (Dim-dip headlights) [1988] ECR 3921

Case 68/86 United Kingdom $v$. Council (Agricultural Hormones) [1988] ECR 855

Cases 89, 91/86 L'Etoile Commerciale v. Commission [1987] ECR 3005

Case 120/86 Mulder $v$. Minister van Landbouw en Visserij [1988] ECR 2321

Case 131/86 United Kingdom $v$. Council (Battery Hens) [1988] ECR 905

Case 201/86 Spie-Batignolles $v$. Commission [1990] ECR I-197 364

Case 222/86 UNECTEF $v$. Heylens [1987] ECR 4097 188, 191, 209, 286

Case 240/86 Commission $v$. Greece [1988] ECR 1835

Case 272/86 Commission v. Greece [1988] ECR 4875

Cases 326/86 and 66/88 Francesconi $v$. Commission [1989] ECR 2087 
Case 186/87 Cowan $v$. Le Trésor public [1989] ECR 195

Case 226/87 Commission $v$. Greece [1988] ECR 3611

Case 242/87 Commission $v$. Council (ERASMUS) [1989] ECR 1425

Case 247/87 Star Fruit v. Commission [1989]

ECR 291

Case 302/87 Parliament v. Council (Comitology) [1988]
ECR 5615
$161,174,204,320,343,346$

Case C-308/87 Grifoni $v$. Euratom [1990] ECR I-1203

352,362

Case 355/87 Commission $v$. Council (Italo-Algerian Maritime Transport) [1989] ECR 1517

Case 374/87 Orkem $v$. Commission [1989] ECR 3283

Case 377/87 Parliament v. Council [1988] ECR 4017

Case C-2/88 Imm Zwartveld [1990] ECR I-3365

Case 5/88 Wachauf $v$. Federal Republic of Germany [1989] ECR 2609

Case C-8/88 Germany $v$. Commission [1990] ECR I-2321

Case 22/88 Vreugdenhil $v$. Minister van Landbouw and Visserij [1989] ECR 2049

Case C-49/88 Al-Jubail Fertiliser v. Council [1991] ECR I-3187 183

Case 68/88 Commission $v$. Greece [1989] ECR 2965

Case C-70/88 Parliament $v$. Council (Chernobyl) [1990] ECR I-2041

Case 100/88 Oyowe and Traore v. Commission [1989] ECR $4285 \quad 190$

Case 103/88 Fratelli Costanzo v. Milano [1989] ECR $1839 \quad 270$

Cases C-143/88 and C-92/89 Zuckerfabrik Süderithmarschen \& Zuckerfabrik Soest [1991] ECR I-415

Case C-150/88 Eau de Cologne v. Provide [1989] ECR 3891

Case C-152/88 Sofrimport Sarl v. Commission [1990] ECR I-2477

$185,328,355-6,358-9,362$

Case C-177/88 Dekker $v$. Stichting Vormingscentrum voor Jong Volwassenen [1990] ECR I-3941 289, 293

Case C-221/88 ECSC v. Acciaierie e ferriere Busseni [1990] ECR I-495

Case C-262/88 Barber $v$. Guardian Royal Exchange [1990] ECR I-1889

Cases C-297/88, 197/89 Dzodzi v. Belgium [1990] ECR I-3763

Case C-322/88 Grimaldi $v$. Fonds des Maladies Professionnelles [1989] ECR 4407 203, 237, 275

Case C-331/88 R v. Minister for Agriculture, Fisheries and Food, ex parte Fedesa [1990] ECR 4023

Case 353/88 Briantex $v$. Council and Commission [1989] ECR 3623

Case C-361/88 Commission $v$. Germany [1991] ECR I-2567

Case C-366/88 France $v$. Commission [1990] ECR I-3571

Case C-63/89 Les Assurances du Crédit SA v. Council and Commission [1991] ECR I-1799 
Cases C-104/89 and 37/90 Mulder and Heinemann v. Commission and Council [1992] ECR I-3061

$359,361-2$

Case C-106/89 Marleasing SA v. La Comercial Internacional de

Alimentación [1990] ECR I-4135

Case C-170/89 BEUC v. Commission [1991] ECR I-5709

Case C-188/89 Foster v. British Gas plc [1990] ECR I-3313

Case C-189/89 Spagl [1990] ECR I-4539

Case C-213/89 R v. Secretary of State for Transport, ex parte Factortame Ltd (Factortame I) [1990] ECR I-2433

Case C-221/89 Factortame II [1991] ECR I-3905 $16,226,244,271,288,299$

Case C-231/89 Gmurzynska-Bscher v. Oberfinanzdirektion Köln [1990] ECR I-4003

Case C-234/89 Delimitis $v$. Henninger Bräu AG [1991] ECR I-935 210

Case C-246/89R Commission v. United Kingdom [1989] ECR 3125

Case C-260/89 Elliniki Radiophonia Tileorasi v. Dimotiki Etairia Pliroforissis (ERT) [1991] ECR I-2925

Case C-292/89 R v. Immigration Appeal Tribunal, ex parte Antonissen [1991] ECR I-745

Case C-298/89 Government of Gibraltar v. Council [1993] ECR I-3605

Case C-300/89 Commission $v$. Council (Titanium Dioxide) [1991] ECR I-2867

Case C-309/89 Codorniu v. Council [1994] ECR I-1853

Case C-340/89 Vlassopoulou v. Bundesministerium für Justiz, Baden-Württemberg [1991] ECR I-2357

Case C-358/89 Extramet Industrie SA v. Council [1991] ECR I-2501

Case C-374/89 Commission v. Belgium [1991] ECR I-367

Case C-377/89 Cotter and McDermott $v$. Minister for Social Welfare (No. 2) [1991] ECR I-1155

Cases C-6, 9/90 Francovich $v$. Italian State (Francovich I) [1991] ECR I-5357 $88,188,200,211,263,275,277,279-81,291$, 299-300, 361, 376

Case C-33/90 Commission v. Italy [1991] ECR I-5987

Case C-65/90 Parliament $v$. Council (Cabotage Regulation) [1992] ECR I-4593

Case C-159/90 Society for the Protection of Unborn Children (Ireland) Ltd (SPUC) v. Grogan [1991] ECR I-4685

Case C-186/90 Durighello v. INPS [1991] ECR I-5773

Case C-208/90 Emmott $v$. Minister for Social Welfare [1991] ECR I-4269

Cases C-271, etc./90 Spain et al. v. Commission [1992] ECR I-5833

Case C-282/90 Vreugdenhil v. Commission [1992] ECR I-1937 356, 363 
Case C-294/90 British Aerospace and Rover Group Holdings plc v. Commission [1992] ECR I-493

Case C-295/90 Parliament v. Council (Students' Rights) [1992] ECR I-4193

Case C-303/90 France v. Commission [1991] ECR I-5315

Case C-313/90 CIRFS v. Commission [1993] ECR I-1125

Cases C-320-322/90 Telemarsicabruzzo v. Circostel [1993] ECR I-393

Case C-343/90 Lourenço Dias v. Director da Alfândega do Porto [1992] ECR I-4673

234,236

Cases C-15, 108/91 Buckl v. Commission [1992] ECR I-6061

Case C-25/91 Pesqueras Echebastar v. Commission [1993] ECR I-1719

Case C-83/91 Meilicke v. ADV/ORGA [1992] ECR I-4871

Case C-107/91 ENU v. Commission [1993] ECR I-599

Case C-109/91 Ten Oever $v$. Stichting Bedrijfspensioenfonds [1993] ECR I-4879

Case C-146/91 KYDEP v. Council and Commission [1994] ECR I-4199

Case C-155/91 Commission v. Council (Waste Directive) [1993] ECR I-939

Case C-168/91 Konstantinidis $v$. Stadt Altensteig [1993] ECR I-1191

Cases C-181, 248/91 Parliament $v$. Council and Commission (Aid to Bangladesh) [1993] ECR I-3685

Case C-198/91 William Cook plc $v$. Commission [1993] ECR I-2486

Case C-220/91P Stahlwerke Peine-Salzgitter v. Commission [1993] ECR I-2393

Cases C-267, 268/91 Keck and Mithouard [1993] ECR I-6097

Case C-271/91 Marshall v. Southampton and South West Hampshire AHA (Marshall II) [1993] ECR I-4367

Case C-316/91 Parliament $v$. Council (Lomé Convention) [1994] ECR I-6250

$267-8,289$

Case C-327/91 France v. Commission [1994] ECR I-3641

Case C-338/91 Steenhorst-Neerings $v$. Bestuur van de

Bedrijfsvereniging voor Detailhandel [1993] ECR I-5475

Case C-24/92 Corbiau [1993] ECR I-1277

Case C-41/92 The Liberal Democrats $v$. Parliament [1993] ECR I-3153

Case C-91/92 Faccini Dori [1994] ECR I-3325

Cases C-92, 326/92 Phil Collins v. Imtrat Handelsgesellschaft $\mathrm{mbH}$ [1993] ECR I-5145

Case C-137/92 P Commission $v$. BASF [1994] ECR I-2555 113, 313, 319

Case C-157/92 Pretore di Genova v. Banchero [1993] ECR I-1085 237

Case C-188/92 TWD Textilwerke Deggendorf GmbH $v$. Germany [1994] ECR I-833 
Case C-238/92 Banks \& Co. Ltd. v. British Coal Corporation [1994] ECR I-1209

Case C-334/92 Wagner Miret $v$. Fondo de Garantía Salarial [1993] ECR I-6911

Case C-359/92 Germany v. Council (Product Safety Directive)

Case C-386/92 Monin Automobiles $v$. France (Monin I) [1993] ECR I-2049

Case C-388/92 Parliament $v$. Council [1994] ECR I-2067

Case C-398/92 Mund \& Fester $v$. Firma Hatrex International Transport [1994] ECR I-467

Case C-404/92 P X v. Commission [1994] ECR I-4780

Case C-405/92 Etablissements A. Mondiet v. Armament Islais [1993] ECR I-6133

Case C-410/92 Johnson v. Chief Adjudication Officer [1994] ECR I-5483

Case C-419/92 Scholz v. Opera Universitaria de Cagliari [1994] ECR 1-505

Case C-422/92 Commission v. Germany (Waste Directives) [1995] ECR I-1097

Case C-431/92 Commission $v$. Germany (Environmental Impact Assessment) [1995] ECR I-2189

Case C-13/93 P v. S. and Cornwall County Council [1996] IRLR 347

Case C-32/93 Webb $v$. EMO Cargo Ltd [1994] ECR I-3567

Case C-41/93 France $v$. Commission (PCP) [1994] ECR I-1829

Cases C-46, 48/93 Brasserie du Pêcheur v. Germany, $\mathrm{R} v$. Secretary of State for Transport, ex parte Factortame Ltd (Factortame III) [1996] 1 CMLR $889 \quad 88,226,279-81,287,291-4$, $299,303,371,376$

Case C-65/93 Parliament $v$. Council (Generalised Tariff Preferences) [1995] ECR I-643

Case C-143/93 Gebroeders van Es Douane Agenten BV $v$. Inspecteur der Invoerrechten en Accijnzen (13.2.96)

Case C-156/93 Parliament $v$. Commission (Micro-organisms) [1995] ECR I-2019

Case C-187/93 Parliament $v$. Council (Transport of Waste) [1994] ECR I-2857

Case C-280/93 Germany $v$. Council (Banana Regulation) [1994] ECR I-4873

Case C-291/93 Commission $v$. Italy [1994] ECR I-859

Case C-312/93 Peterbroeck v. Belgian State [1996] 1 CMLR 793 [1995] ECR I-4599

Case C-316/93 Vaneetveld $v$. SA Le Foyer [1994] ECR I-763

Case C-350/93 Spain $v$. Council (Patent protection for medical products) [1995] ECR I-1985 
Cases C-358, 416/93 Bordessa [1995] ECR I-361

Case C-392/93 R v. HM Treasury, ex parte British Telecom

$279,281,292$

Case C-412/93 Leclerc-Siplec $v$. TFI Publicité [1995] ECR I-179 235

Case C-415/93 Union Royale belge des Sociétés de football association ASBL v. Bosman [1996] 1 CMLR 645 [1995] ECR I-4921

Case C-417/93 Parliament $v$. Council (TACIS) [1995] ECR I-1185 146

Case C-426/93 Germany v. Council (Statistical Registers) [1995] ECR I-3723

Case C-428/93 Monin Automobiles $v$. France (Monin II) [1994] ECR I-1707

Cases C-430, 431/93 Van Schijndel $v$. Stichting Pensioenfonds voor Fysiotherapeuten [1996] 1 CMLR 801 [1995] ECR I-4705 290-1

Case C-465/93 Atlanta Fruchthandelsgesellschaft mbH $v$. Bundesamt für Ernährung und Forstwirtschaft [1995] ECR I-3761

Case C-479/93 Francovich $v$. Italian Republic (Francovich II) [1995] ECR I-3843

Case C-21/94 Parliament v. Council (Harmonized Road Taxes) [1995] ECR I-1827

Case C-25/94 Commission v.Council (FAO) (19.3.96)

$123,171,173,203,319$

Case 58/94 Netherlands $v$. Council (30.4.96) 71,198

Case C-120/94R Commission v. Greece (FYROM) [1994] ECR I-3037

218,221

Cases C-163 etc./94, Sanz de Lera [1996] 1 CMLR 631 [1995] ECR I-4821

Case C-167/94 Grau Gomis [1995] ECR I-1023

265

99

Case C-193/94 Skanavi and Chryssanthakopoulos (29.2.1996)

74

Case C-266/94 Commission v. Spain [1995] ECR I-1975

216

Case C-271/94 Parliament v. Council (Edicom) (26.3.96)

169,176

Case C-41/95 Council v. Parliament [1995] ECR I-4411

167,172

\section{Alphabetical list}

Adams v. Commission Case 145/83 [1985] ECR 3539

352,362

Alcan v. Commission Case 69/69 [1970] ECR 385

Al-Jubail Fertiliser $v$. Council Case C-49/88 [1991] ECR I-3187

Allied Corporation $v$. Commission Cases 239, 275/82 [1984]

ECR 1005

329,332

Alusuisse v. Council and Commission Case 307/81 [1982]

ECR 3463

A.M. \& S. v. Commission Case 155/79 [1982] ECR 1575 
Amministratzione delle Finanze dello Stato v. San Giorgio Case 199/82 [1983] ECR 3595

Amministrazione delle Finanze dello Stato $v$. Simmenthal

(Simmenthal II) Case 106/77 [1978] ECR 629

$40,221,257,270$, 275,287

Amylum v. Council Case 108/81 [1982] ECR 3107 185

Amylum and Tunnel Refineries $v$. Council and Commission

Cases 116, 124/77 [1979] ECR 3479

Atlanta Fruchthandelsgesellschaft $\mathrm{mbH} v$. Bundesamt für

Ernährung und Forstwirtschaft Case C-465/93 [1995]

ECR I-3761

Banks \& Co. Ltd. v. British Coal Corporation Case C-238/92 [1994] ECR I-1209

Barber v. Guardian Royal Exchange Case C-262/88 [1990] ECR I-1889

BAT and Reynolds $v$. Commission (Philip Morris) Cases 142 and 156/84 [1987] ECR 4487

Bayerische HNL et al. v. Council and Commission (Second Skimmed Milk Powder case) Cases 83, etc./76 [1978] ECR 1209 356-7

Beauport v. Council and Commission Cases 103-9/78 [1979] ECR 17

Becker Case 8/81 [1982] ECR 53

Bela Mühle Josef Bergman v. Grows-Farm (Skimmed Milk Powder) Case 114/76 [1977] ECR 1211

Bethell v. Commission Case 246/81 [1982] ECR 2277

BEUC v. Commission Case C-170/89 [1991] ECR I-5709

Bock v. Commission Case 62/70 [1971] ECR 897

Bordessa Cases C-358, 416/93 [1995] ECR I-361

Bosch $v$. de Geus [1962] ECR 45 Case 13/61

Brasserie du Pêcheur $v$. Germany, R. v. Secretary of State for Transport, ex parte Factortame Ltd (Factortame III) Cases C-46, 48/93 [1996] 1 CMLR 889 $88,226,279-81,287,291-4$, $299,303,371,376$

Briantex v. Council and Commission Case 353/88 [1989] ECR 3623

British Aerospace and Rover Group Holdings plc $v$. Commission Case C-294/90 [1992] ECR I-493

Calpak SpA v. Commission Cases 789-790/79 [1980] ECR 1949

Casagrande $v$. Landeshauptstadt München Case 9/74 [1974] ECR 773 
Cotter and McDermott $v$. Minister for Social Welfare (No. 2) Case C-377/89 [1991] ECR I-1155

Chevalley v. Commission Case 15/70 [1970] ECR 975

CILFIT Case 283/81 [1982] ECR 3415

Cimenteries $v$. Commission (Noordwijks Cement Accoord) Cases 8-11/66 [1967] ECR 75

Cinéthèque $v$. Fédération nationale des cinémas français Cases 60-1/84 [1985] ECR 2605

CIRFS v. Commission Case C-313/90 [1993] ECR I-1125

CNTA v. Commission Case 74/74 [1975] ECR 533

$184,356,361$

Codorniu v. Council Case C-309/89 [1994] ECR I-1853

$328-30$

COFAZ v. Commission Case 169/84 [1986] ECR 391

Comet $v$. Produktschap Case 45/76 [1976] ECR 2043

Commission v. BASF Case C-137/92 P [1994] ECR I-2555 113, 313, 319

Commission $v$. Belgium Case 156/77 [1978] ECR 1881

Commission $v$. Belgium Case 85/85 [1986] ECR 1149

217,319

Commission v. Belgium Case 293/85 [1988] ECR 305

Commission $v$. Belgium Case C-374/89 [1991] ECR I-367

208

Commission $v$. Belgium (Pressed Wood) Case 77/69 [1970] ECR 237

Commission v. Belgium (Water Pollution) Case 1/86 [1987] ECR 2797

Commission $v$. Council (ERASMUS) Case 242/87 [1989]

ECR 1425

Commission $v$. Council (ERTA) Case 22/70 [1971] ECR 263

Commission $v$. Council (FAO) Case C-25/94 (19.3.96)

Commission $v$. Council (Generalised Tariff Preferences) Case 45/ 86 [1987] ECR 1493

Commission $v$. Council (Italo-Algerian Maritime Transport) Case 355/87 [1989] ECR 1517

Commission $v$. Council (Titanium Dioxide) Case C-300/89 [1991] ECR I-2867

Commission $v$. Council (Waste Directive) Case C-155/91 [1993] ECR I-939

Commission v. Denmark Case 211/81 [1982] ECR 4547

Commission $v$. France Case 322/82 [1983] ECR 3705

Commission $v$. France (Euratom Supply Agency) Case 7/71 [1971] ECR 1003

Commission v. France (French Merchant Seamen) Case 167/73 [1974] ECR 359)

Commission v. France (Lamb Wars) Case 232/78 [1979] ECR 2729

Commission v. Germany Case 178/84 [1987] ECR 1227 
Commission v. Germany (Brennwein) Case 24/62 [1963] ECR 63

142,335

Commission $v$. Germany (Environmental Impact Assessment) Case C-431/92 [1995] ECR I-2189

Commission $v$. Germany (Waste Directives) Case C-422/92 [1995] ECR I-1097

Commission v. Greece Case 240/86 [1988] ECR 1835

Commission v. Greece Case 272/86 [1988] ECR 4875

Commission v. Greece Case 226/87 [1988] ECR 3611

Commission v. Greece Case 68/88 [1989] ECR 2965

Commission $v$. Greece (FYROM) Case C-120/94 R [1994] ECR I-3037

Commission $v$. Ireland (Buy Irish) Case 249/81 [1982] ECR $4005 \quad 213$

Commission $v$. Ireland (Fisheries) Case 61/77 R [1977] ECR $937 \quad 220$

Commission $v$. Ireland (Imports of poultry) Case 74/82 [1984] ECR 317

Commission $v$. Ireland (Potatoes) Case 288/83 [1985] ECR 1761

Commission $v$. Italy Case 136/81 [1982] ECR 3547

217

Commission $v$. Italy Case C-33/90 [1991] ECR I-5987

209

Commission $v$. Italy Case C-291/93 [1994] ECR I-859 221

Commission $v$. Italy (Art Treasures II) Case 48/71 [1972] ECR $527 \quad 216$

Commission $v$. Italy (Export Rebates) Case 31/69 [1970] ECR $25 \quad 214$

Commission $v$. Luxembourg and Belgium (Dairy Products) Cases 90 and $91 / 63$ [1964] ECR 625

Commission v. Netherlands Case 96/81 [1982] ECR 1791

Commission v. Spain Case C-266/94 [1995] ECR I-1975

216

Commission v. United Kingdom Case C-246/89 R [1989] ECR 3125

Commission $v$. UK (Dim-dip headlights) Case 60/86 [1988] ECR 3921

Commission $v$. United Kingdom (Pig Producers) Cases 31 and 53/77 R [1977] ECR 921

Compagnie d'Approvisionnement v. Commission Cases 9, 11/71 [1972] ECR 391

Confédération Nationale des Producteurs de Fruits et Légumes $v$. Council Cases 16 and 17/62 [1962] ECR 471

Corbiau Case C-24/92 [1993] ECR I-1277

Costa v. ENEL Case 6/64 [1964] ECR 585

Council v. Parliament Case 34/86 [1986] ECR 2155

167,316

Council v. European Parliament Case C-41/95 [1995] ECR I-4411 
Defrenne $v$. SABENA Case 43/75 [1976] ECR 455

Defrenne $v$. SABENA Case 149/77 [1978] ECR 1365

Dekker $v$. Stichting Vormingscentrum voor Jong Volwassenen Case C-177/88 [1990] ECR I-3941

Delimitis $v$. Henninger Bräu AG Case C-234/89 [1991] ECR I-935 210

Demirel v. Stadt Schwäbisch Gmund Case 12/86 [1987] ECR $3719 \quad 237$

Denkavit Italiana Case 61/79 [1980] ECR 1205

Deutsche Grammophon v. Metro-SB-Großmärkte Case 78/70 [1971] ECR 487

Deutsche Milchkontor GmbH v. Germany Cases 205-215/82 [1983] ECR 2633

Donckerwolcke $v$. Procureur de la République Case 41/76 [1976] ECR 1921

Dumortier et al. v. Council and Commission (Quellmehl and Gritz) Cases 64, etc./76 [1979] ECR 3091

Durighello $v$. INPS Case C-186/90 [1991] ECR I-5773

Dzodzi v. Belgium Cases C-297/88 and C-197/89 [1990] ECR I-3763

Eau de Cologne v. Provide Case C-150/88 [1989] ECR 3891

ECSC v. Acciaierie e ferriere Busseni Case C-221/88 [1990] ECR I-495

Einfuhr- und Vorratstelle $v$. Köster Case 25/70 [1970] ECR 1161

Electroimpex $v$. Council Case C-157/87 [1990] ECR I-3021

Elliniki Radiophonia Tileorasi $v$. Dimotiki Etairia Pliroforissis (ERT) Case C-260/89 [1991] ECR I-2925

Emmott $v$. Minister for Social Welfare Case C-208/90 [1991] ECR I-4269

ENU v. Commission Case C-107/91 [1993] ECR I-599

Etablissements A. Mondiet v. Armament Islais Case C-405/92 [1993] ECR I-6133

Faccini Dori Case C-91/92 [1994] ECR I-3325

Extramet Industrie SA $v$. Council Case C-358/89 [1991]

ECR I-2501

Fédéchar $v$. High Authority Case 8/55 [1956] ECR 292

FEDIOL v. Commission Case 191/82 [1983] ECR 2913

Firma Foto-Frost $v$. Hauptzollamt Lübeck Case 314/85 [1987] ECR 4199

Firma Schwarze $v$. Einfuhr- und Vorratstelle für Getreide und Futtermittel Case 16/65 [1965] ECR 877

Foglia v. Novello (No. 1) Case 104/79 [1980] ECR 745

Foglia $v$. Novello (No. 2) Case 244/80 [1981] ECR 3045

Ford of Europe Inc $v$. Commission Cases 228, 229/82 [1984] ECR 1129

Foster v. British Gas plc Case C-188/89 [1990] ECR I-3313 
France v. Commission Case C-366/88 [1990] ECR I-3571

France v. Commission Case C-303/90 [1991] ECR I-5315

France v. Commission Case C-327/91 [1994] ECR I-3641

France v. Commission (PCP) Case C-41/93 [1994] ECR I-1829

France v. United Kingdom Case 141/78 [1979] ECR 2923

Francesconi $v$. Commission Cases 326/86 and 66/88 [1989] ECR 2087

Francovich $v$. Italian State (Francovich I) Cases C-6, 9/90 [1991] ECR I-5357

$88,188,200,211,263,275,277,279-81$, $291,299-300,361,376$

Francovich $v$. Italian Republic (Francovich II) Case C-479/93 [1995] ECR I-3843

Fratelli Costanzo v. Milano Case 103/88 [1989] ECR 1839

GAEC v. Council and Commission Case 253/84 [1987] ECR $123 \quad 361$

GEMA v. Commission Case 125/78 [1979] ECR 3173

Gebroeders van Es Douane Agenten BV v. Inspecteur der

Invoerrechten en Accijnzen Case C-143/93 (13.2.96) 184

Germany v. Commission Case C-8/88 [1990] ECR I-2321 209

Germany et al. v. Commission (Migration Policy)

Case 281, etc./85 [1987] ECR 3203

$81,115,142,201$

Germany v. Council (Banana Regulation) Case C-280/93 [1994] ECR I-4873

93,309

Germany $v$. Council (Product Safety Directive) Case C-359/92 [1994] ECR I-3681

Germany v. Council (Statistical Registers) Case C-426/93 [1995] ECR I-3723

Germany $v$. High Authority Case 3/59 [1960] ECR 53

Gmurzynska-Bscher $v$. Oberfinanzdirektion Köln Case C-231/89 [1990] ECR I-4003

Government of Gibraltar v. Council Case C-298/89 [1993] ECR I-3605

Grau Gomis Case C-167/94 [1995] ECR I-1023

Gravier v. City of Liège Case 293/83 [1985] ECR 593

Grifoni $v$. Euratom Case C-308/87 [1990] ECR I-1203

Grimaldi $v$. Fonds des Maladies Professionnelles Case C-322/88

[1989] ECR 4407

Haegemann $v$. Commission Case 96/71 [1972] ECR 1005

Hauer v. Land Rheinland Pfalz Case 44/79 [1979] ECR 3740

Hauptzollamt Bremerhaven v. Massey-Ferguson Case 8/73 [1973] ECR 897

Hoechst v. Commission Case 46/87 [1989] ECR 2859

Hoffmann-La-Roche $v$. Centrafarm Case 107/76 [1977] ECR $957 \quad 242$

Humblet $v$. Belgium Case 6/60 [1960] ECR 559

Hurd v. Jones Case 44/84 [1986] ECR 29 
IBM v. Commission Case 60/81 [1981] ECR 2639

Ilford v. Commission Case 1/84R [1984] ECR 423

International Chemical Corporation $v$. Amministrazione delle

Finanze dello Stato Case 66/80 [1981] ECR 1191

$242,245-6$

International Fruit Company v. Commission Cases 41-44/70

[1971] ECR 411

$199,326,328$

International Fruit Company NV v. Produktschap voor

Groenten en Fruit Cases 2-4/72 [1972] ECR 1219

93,265

Internationale Handelsgesellschaft Case 11/70 [1970]

ECR 1125

$189-90,258$

Irish Creamery Milk Suppliers Association v. Ireland Cases 36, $71 / 80$ [1981] ECR 735

Italy v. Commission Case 32/65 [1966] ECR 389

Johnson v. Chief Adjudication Officer Case C-410/92 [1994]

ECR I-5483

Johnston $v$. Chief Constable of the Royal Ulster Constabulary

Case 222/84 [1986] ECR 1651

$86,188,267,270,276,286,329$

Kampffmeyer $v$. Commission Cases 5, etc./66 [1967] ECR 245

Keck and Mithouard Cases C-267, 268/91 [1993] ECR I-6097

Kolpinghuis Nijmegen Case 80/86 [1987] ECR 3969

Konstantinidis v. Stadt Altensteig Case C-168/91 [1993]

ECR I-1191

Kramer Cases 3/76 etc. [1976] ECR 1279

75,193

Krohn $v$. Commission Case 175/84 [1986] ECR 753

KSH v. Commission Case 101/76 [1977] ECR 797

KSH NV v. Council and Commission Case 143/77 [1979]

ECR 3583

Kupferberg Case 104/81 [1982] ECR 3659

KYDEP v. Council and Commission Case C-146/91 [1994]

ECR I-4199

Lassalle v. Parliament Case 15/63 [1964] ECR 31

Leclerc-Siplec $v$. TFI Publicité Case C-412/93 [1995] ECR I-179

Les Assurances du Crédit SA v. Council and Commission Case C-63/89 [1991] ECR I-1799

L'Etoile Commerciale v. Commission Cases 89, 91/86 [1987] ECR 3005

Lourenço Dias v. Director da Alfândega do Porto Case C-343/90 [1992] ECR I-4673

Lütticke v. Commission Case 48/65 [1966] ECR 19

234,236

Lütticke v. Commission Case 4/69 [1971] ECR 325

215,348

Lütticke $v$. HZA Saarlouis Case 57/65 [1966] ECR 205

Luxembourg v. Parliament Case 230/81 [1983] ECR 255

202,315 
Marleasing SA $v$. La Comercial Internacional de Alimentación

Case C-106/89 [1990] ECR I-4135

$274-6,297$

Marshall $v$. Southampton and South West Hampshire AHA

(Marshall I) Case 152/84 [1986] ECR 723

Marshall $v$. Southampton and South West Hampshire AHA

(Marshall II) Case C-271/91 [1993] ECR I-4367

$267-8,289$

Meilicke v. ADV/ORGA Case C-83/91 [1992] ECR I-4871

Meroni v. High Authority Case 9/56 [1957-58] ECR 133

Metro-SB-Großmärkte v. Commission Case 26/76 [1977] ECR 1875

Milch- Fett- und Eierkontor v. HZA Saarbrücken Case 29/68 [1969] ECR 165

Molkerei Zentrale Westfalen $v$. HZA Paderborn Case 28/67 [1968] ECR 143

Monin Automobiles $v$. France (Monin I) Case C-386/92 [1993] ECR I-2049

Monin Automobiles $v$. France (Monin II) Case C-428/93 [1994] ECR I-1707

Mulder $v$. Minister van Landbouw en Visserij Case 120/86 [1988] ECR 2321

$185,336,359$

Mulder and Heinemann $v$. Commission and Council Cases C-104/89 and 37/90 [1992] ECR I-3061

Mund \& Fester $v$. Firma Hatrex Internationaal Transport Case

C-398/92 [1994] ECR I-467

Municipality of Differdange $v$. Commission Case 222/83 [1984] ECR 2889

Netherlands $v$. Commission Case 59/70 [1971] ECR 639

Netherlands $v$. Council Case C-58/94 (30.4.96)

Nold v. Commission Case 4/73 [1974] ECR 503

Nordgetreide $v$. Commission Case 42/71 [1972] ECR 105

Nordsee $v$. Reederei Mond Case 102/81 [1982] ECR 1095

NTN Toyo Bearing Co v. Commission Case 113/77 R [1977] ECR 1721

NTN Toyo Bearing Co v. Council and Commission Case 113/77 [1979] ECR 1185

Nuovo Campsider v. Commission Case 25/85 [1986] ECR 1531

Orkem v. Commission Case 374/87 [1989] ECR 3283

P v. S and Cornwall County Council Case C-13/93 [1996] IRLR 347

Parliament $v$. Commission (Micro-organisms) Case C-156/93 [1995] ECR I-2019

Parliament $v$. Council Case 377/87 [1988] ECR 4017 
Parliament $v$. Council and Commission (Aid to Bangladesh)

Cases C-181, 248/91 [1993] ECR I-3685

Parliament $v$. Council (Cabotage Regulation) Case C-65/90

[1992] ECR I-4593

Parliament $v$. Council (Chernobyl) Case C-70/88 [1990]

ECR I-2041

$70,174,204,320$

Parliament v. Council (Comitology) Case 302/87 [1988]

ECR 5615

$161,174,204,320,343,345$

Parliament $v$. Council (Edicom) Case C-271/94 (26.3.96)

169,176

Parliament $v$. Council (Generalised Tariff Preferences) Case

C-65/93 [1995] ECR I-643

146

Parliament $v$. Council (Harmonized Road Taxes) Case C-21/94 [1995] ECR I-1827

Parliament v. Council (Lomé Convention) Case C-316/91 [1994] ECR I-6250

Parliament $v$. Council (Students' Rights) Case C-295/90 [1992] ECR I-4193

Parliament $v$. Council (TACIS) Case C-417/93 [1995] ECR I-1185 146

Parliament $v$. Council (Transport of Waste) Case C-187/93 [1994] ECR I-2857

Parliament $v$. Council (Transport Policy) Case 13/83 [1985]

ECR 1513

$119,173,311,345-6$

Parti Ecologiste 'Les Verts' v. European Parliament Case 294/83 [1986] ECR 1339 $16,87,174,202,303,315-16$, $320,326,337,339,348$

Pesqueras Echebastar v. Commission Case C-25/91 [1993] ECR I-1719

Peterbroeck $v$. Belgian State Case C-312/93 [1996] 1 CMLR 793 [1995] ECR I-4599

Phil Collins v. Imtrat Handelsgesellschaft mbH Cases C-92, 326/ 92 [1993] ECR I-5145

Philip Morris Holland BV v. Commission Case 730/79 [1980] ECR 2671

Pinna v. Caisse d'allocations familiales de la Savoie Case 41/84 [1986] ECR 1

Piraiki-Patraiki v. Commission Case 11/82 [1985] ECR 207

Plaumann v. Commission Case 25/62 [1963] ECR 95

Politi v. Italian Minister of Finance Case 43/71 [1971] ECR 1039

Prantl Case 16/83 [1984] ECR 1299

Pretore di Genova $v$. Banchero Case C-157/92 [1993] ECR I-1085

Pretore di Salò v. X. Case 14/86 [1987] ECR 2545

Procureur de la République $v$. Association de défense des brûleurs d'huiles usagées Case 240/83 [1985] ECR 520

Procureur de la République $v$. Waterkeyn Cases 314/81, etc. [1982] ECR 4337 
R v. HM Treasury, ex parte British Telecom Case C-392/93 (26.3.96) $279,281,292$

R v. Immigration Appeal Tribunal, ex parte Antonissen Case C-292/89 [1991] ECR I-745

R v. Intervention Board for Agricultural Produce, ex parte Man (Sugar) Ltd Case 181/84 [1985] ECR 2889

R v. Minister for Agriculture, Fisheries and Food, ex parte Fedesa Case C-331/88 [1990] ECR 4023

$\mathrm{R} v$. Secretary of State for Home Affairs, ex parte Santillo Case 131/79 [1980] ECR 1585

$\mathrm{R} v$. Secretary of State for Transport, ex parte Factortame Ltd (Factortame I) Case C-213/89 [1990] ECR I-2433 16, 226, 244, 271, 288,299

$\mathrm{R} v$. Secretary of State for Transport, ex parte Factortame Ltd (Factortame II) Case C-221/89 [1991] ECR I-3905

$\mathrm{R} v$. Tymen Case 269/80 [1981] ECR 3079

Ratti Case 148/78 [1979] ECR 1629

Rewe-Handelsgesellschaft Nord $\mathrm{mbH} v$. Hauptzollamt Kiel Case 158/80 [1981] ECR 1805

Rewe-Zentrale AG $v$. Bundesmonopolverwaltung für Branntwein (Cassis de Dijon) Case 120/78 [1979] ECR 649

Rewe-Zentralfinanz $v$. Landwirtschaftskammer für das Saarland Case 33/76 [1976] ECR 1989

Rey Soda $v$. Cassa Conguaglio Zucchero Case 23/75 [1975] ECR 1279

Rheinmühlen-Düsseldorf $v$. Einfuhr- und Vorratstelle für Getreide und Futtermittel Case 166/73 [1974] ECR 33

Richez-Parise v. Commission Cases 19, etc./69 [1970] ECR 325

Roquette v. Commission Case 26/74 [1976] ECR 677

Roquette Frères $v$. Council Case 138/79 [1980] ECR 3333

Royal Scholten-Honig (Holdings) Ltd $v$. Intervention Board for Agricultural Produce Cases 103, 145/77 [1978] ECR $2037 \quad 327,356$

Ruckdeschel $v$. HZA Hamburg St-Annen Cases 117, etc./76 [1977] ECR 1753

Russo v. AIMA Case 60/75 [1976] ECR 45

Rutili v. Minister for the Interior Case 36/75 [1975] ECR 1219

Sanz de Lera Cases C-163 etc. /94 [1996] 1 CMLR 631 [1995] ECR I-4821

Sayag $v$. Leduc Case 9/69 [1969] ECR 329

Scholz v. Opera Universitaria di Cagliari Case C-419/92 [1994] ECR I-505

SFEI $v$. Commission Case C-39/93 P [1994] ECR I-2681 
Société des Usines à Tubes de la Sarre $v$. High Authority Cases 1, 14/57 [1957] ECR 105

Society for the Protection of Unborn Children (Ireland) Ltd

(SPUC) v. Grogan Case C-159/90 [1991] ECR I-4685

Sofrimport $v$. Commission Case C-152/88 [1990]

ECR I-2477

$185,328,355-6,358-9,362$

Spagl Case C-189/89 [1990] ECR I-4539

336,359

Spain et al. v. Commission Cases C-271, etc./90 [1992]

ECR I-5833

Spain v. Council (Patent protection for medical products) Case

C-350/93 [1995] ECR I-1985

SPI Cases 267-269/81 [1983] ECR 801

Spie-Batignolles $v$. Commission Case 201/86 [1990] ECR I-197

Spijker Kwasten $v$. Commission Case 231/82 [1983] ECR 2559

Stahlwerke Peine-Salzgitter $v$. Commission Case C-220/91P [1993] ECR I-2393

Star Fruit v. Commission Case 247/87 [1989]

ECR 291

Stauder v. City of Ulm Case 29/69 [1969] ECR 419

Steenhorst-Neerings $v$. Besuur van de Bedrijfsvereniging voor

Detailhandel Case C-338/91 [1993] ECR I-5475

Telemarsicabruzzo v. Circostel Cases C-320-322/90 [1993]

ECR I-393

Ten Oever $v$. Stichting Bedrijfspensioenfonds Case C-109/91 [1993] ECR I-4879

The Liberal Democrats $v$. Parliament Case C-41/92 [1993] ECR I-3153

Timex v. Council and Commission Case 264/82 [1985] ECR 849

Toepfer v. Commission Cases 106-107/63 [1965] ECR $405 \quad 323,360$

Transocean Marine Paint Association v. Commission Case 17/74 [1974] ECR 1063

TWD Textilwerke Deggendorf GmbH v. Germany Case C-188/ 92 [1994] ECR I-833

UNECTEF $v$. Heylens Case 222/86 [1987] ECR 4097 188, 191, 209, 286

Union Deutsche Lebensmittelwerke $v$. Commission Case 97/85 [1987] ECR 2265

Union Royale belge des Sociétés de football association ASBL $v$. Bosman Case C-415/93 [1996] 1 CMLR 645 [1995] ECR I-4921

United Kingdom $v$. Council (Agricultural Hormones) Case 68/86 [1988] ECR 855

United Kingdom $v$. Council (Battery Hens) Case 131/86 [1988] ECR 905

Universität Hamburg v. HZA Hamburg-Kehrwieder Case 216/82 [1983] ECR 2771 
Van Duyn v. Home Office Case 41/74 [1974] ECR 1337

$40,265-6$

Van Gend en Loos $v$. Nederlandse Administratie der Belastingen

Case 26/62 [1963] ECR 1 $34,247,252,262-3,283$

Van Schijndel $v$. Stichting Pensioenfonds voor Fysiotherapeuten

Cases C-430, 431/93 [1996] 1 CMLR 801 [1995] ECR I-4705 290-1

Vaneetveld $v$. SA Le Foyer Case C-316/93 [1994] ECR I-763 268

Variola $v$. Italian Finance Administration Case 34/73 [1973]

ECR 981

Vlassopoulou $v$. Bundesministerium für Justiz,

Baden-Württemberg Case C-340/89 [1991] ECR I-2357

Von Colson and Kamann v. Land Nordrhein Westfalen Case

14/83 [1984] ECR 1891 209, 267, 272-4, 289

Vreugdenhil $v$. Commission Case C-282/90 [1992] ECR I-1937 356, 363

Vreugdenhil v. Minister van Landbouw and Visserij Case 22/88 [1989] ECR 2049

Wachauf $v$. Federal Republic of Germany Case 5/88 [1989] ECR 2609

Wagner Miret $v$. Fondo de Garantía Salarial Case C-334/92 [1993] ECR I-6911

Walter Rau v. BALM Case 133-6/85 [1987] ECR 2289

Webb $v$. EMO Cargo Ltd Case C-32/93 [1994] ECR I-3567

William Cook plc $v$. Commission Case C-198/91 [1993]

ECR I-2486

Wöhrmann v. Commission Cases 31, 33/62 [1962] ECR 501

X v. Commission Case C-404/92 P [1994] ECR I-4780

Zuckerfabrik Schöppenstedt $v$. Council Case 5/71 [1971] ECR 975

Zuckerfabrik Süderithmarschen \& Zuckerfabrik Soest Cases

C-143/88 and C-92/89 [1991] ECR I-415

Zwartveld, Imm Case C-2/88 [1990] ECR I-3365

\section{Opinions delivered pursuant to Article 228 EC}

Opinion 1/75 Re the Draft Understanding on a Local Cost Standard [1975] ECR 1355

Opinion $1 / 91 \mathrm{Re}$ the Draft Agreement on a European Economic Area [1991] ECR I-6079 $95,98-9,197,256$

Opinion 2/91 Re ILO Convention [1993] ECR I-1061 $81,91-2$

Opinion 1/92 Re the Draft Agreement on a European Economic Area (No. 2) [1992] ECR I-2821 


\section{B: Cases before the Court of First Instance}

\section{By Case Number (in year order)}

Case T-7/89 Hercules $v$. Commission (Polypropylene) [1991] ECR I-1711

Case T-56/89 R Publishers Association v. Commission [1989] ECR 1693

Case T-64/89 Automec v. Commission (Automec I) [1990] ECR II-367

Cases T-79 etc./89 BASF v. Commission [1992] ECR II-315 112, 319

Case T-120/89 Stahlwerke Peine-Salzgitter v. Commission [1991] ECR II-279

Case T-24/90 Automec $v$. Commission (Automec II) [1992] ECR II-2223

Case T-30/91 Solvay v. Commission [1995] ECR II-1775

Case T-29/92 SPO v. Commission [1995] ECR II-289

Case T-37/92 BEUC $v$. Commission [1994] ECR II-285

Case T-83/92 Zunis Holdings SA v. Commission [1993] ECR II-1169

Case T-96/92 Comité central d'entreprise de la Société générale des grande sources $v$. Commission [1995] ECR II-1213

Case T-3/93 Société Anonyme à Participation Ouvrière Nationale Air France v. Commission (Air France) [1994] ECR II-121

Case T-12/93 Comité central d'entreprise de la Société anonyme Vittel $v$. Commission [1995] ECR II-1247

Case T-466/93 etc. O’Dwyer v. Council [1995] ECR II-2071 185

Case T-472/93 Campo Ebro Industrial $v$. Council [1995] ECR II-421

Case T-476/93 FRSEA and FNSEA $v$. Council [1993] ECR II-1187

Case T-490/93 Bremer Vulkan Verbund AG v. Commission [1995] ECR II-477

Case T-572/93 Odigitria AAE $v$. Council and Commission [1995] ECR II-2025

Case T-584/93 Roujansky v. European Council [1994] ECR II-585

Case T-585/93 Stichting Greenpeace Council v. Commission [1995] ECR II-2205

Case T-84/94 Bundesverband der Bilanzbuchhalter eV $v$. Commission [1995] ECR II-101

Case T-88/94 Société Commerciale des Potasses et de l'Azote $v$. Commission [1995] ECR II-222

Case T-95/94 Chambre Syndicale Nationale des Enterprises de Transport de Fonds et Valeurs and Brink's France Sarl $v$. Commission (28.9.95) 
Case T-107/94 Kik v. Council and Commission [1995] ECR II-1717

Case T-117/94 Associazione Agricoltori della Provincia di

Rovigo et al. v. Commission (Po Delta) [1995] ECR II-455

Case T-186/94 Guérin Automobiles v. Commission [1995] ECR II-1753

Case T-194/94 Carvel and Guardian Newspapers $v$. Council

[1995] 3 CMLR 359

$71,76,119,198,311$

Case T-219/95R Danielsson v. Commission (22.12.95)

\section{Alphabetical list}

Associazione Agricoltori della Provincia di Rovigo et al. $v$. Commission (Po Delta) Case T-117/94 [1995] ECR II-455

Automec v. Commission (Automec I) Case T-64/89 [1990] ECR II-367

86,318

Automec v. Commission (Automec II) Case T-24/90 [1992] ECR II-2223

BASF v. Commission Cases T-79/89, etc. [1992] ECR II-315

BEUC v. Commission Case T-37/92 [1994] ECR II-285

Bremer Vulkan Verbund AG $v$. Commission Case T-490/93 [1995] ECR II-477

Bundesverband der Bilanzbuchhalter eV v. Commission Case T-84/94 [1995] ECR II-101

Campo Ebro Industrial v. Council Case T-472/93 [1995] ECR II-421

Carvel and Guardian Newspapers $v$. Council Case T-194/94 [1995] 3 CMLR 359 $71,76,119,198,311$

Chambre Syndicale Nationale des Enterprises de Transport de Fonds et Valeurs and Brink's France Sarl $v$. Commission Case T-95/94 (28.9.95)

Comité central d'entreprise de la Société anonyme Vittel $v$. Commission Case T-12/93 [1995] ECR II-1247

Comité central d'entreprise de la Société générale des grande sources $v$. Commission Case T-96/92 [1995] ECR II-1213

Danielsson $v$. Commission Case T-219/95R (22.12.95)

FRSEA and FNSEA $v$. Council Case T-476/93 [1993] ECR II-1187

Guérin Automobiles $v$. Commission Case T-186/94 [1995] ECR II-1753

Hercules $v$. Commission (Polypropylene) Case T-7/89 [1991] ECR I-1711

Kik v. Council and Commission Case T-107/94 [1995] ECR II-1717

O'Dwyer v. Council Case T-466/93 etc. [1995] ECR II-2071 185

Odigitria AAE v. Council and Commission Case T-572/93 [1995] ECR II-2025 
Publishers Association v. Commission Case T-56/89 R [1989] ECR 1693

Roujansky v. European Council Case T-584/93 [1994]

ECR II-585

Société Anonyme à Participation Ouvrière Nationale Air France

v. Commission (Air France) Case T-3/93 [1994]

ECR II-121

$201,223,318,331$

Société Commerciale des Potasses et de l'Azote $v$. Commission

Case T-88/94 [1995] ECR II-222

Solvay v. Commission Case T-30/91 [1995] ECR II-1775 183

SPO v. Commission Case T-29/92 [1995] ECR II-289

Stahlwerke Peine-Salzgitter $v$. Commission Case T-120/89 [1991]

ECR II-279

351,358

Stichting Greenpeace Council v. Commission Case T-585/93

[1995] ECR II-2205

325

Zunis Holdings SA $v$. Commission Case T-83/92 [1993]

ECR II-1169

\section{C: Cases before National Courts}

\section{English Courts}

Blackburn $v$. Attorney General [1971] 1 All ER 1380

Bourgoin v. MAFF [1986] QB $716 \quad 298$

British Airways $v$. Laker Airways [1984] 3 All ER 39

Bulk Oil $v$. Sun International ([1984] 1 WLR 147

Bulmer $v$. Bollinger [1974] Ch. 401; [1974] 2 All ER 1226

Chiron v.Murex [1994] FSR 187 (CA); [1995] All ER (EC) 88 (HL)

Commissioners of Customs and Excise v. Samex ApS [1983] 3 CMLR 194

Duke v. GEC Reliance [1988] 1 All ER 626

Garden Cottage Foods v. Milk Marketing Board [1984] $\mathrm{AC} 130$

Kirklees BC $v$. Wickes Building Supplies Ltd [1992] 3 All ER $717 \quad 299$

Litster $v$. Forth Dry Dock and Engineering Co Ltd [1989] 1 All ER 1134

Macarthys $v$. Smith [1979] 3 All ER 325

Pickstone $v$. Freemans plc [1988] 2 All ER 813

R $v$. Henn and Darby [1978] 3 All ER 1190 (CA); [1980] 2 All ER 166 (HL)

$\mathrm{R} v$. International Stock Exchange of the United Kingdom and the Republic of Ireland, ex parte Else [1993] 1 All ER 420

R v. London Boroughs' Transport Committee, ex parte Freight Transport Association [1991] 3 All ER 915 
R v. Secretary of State for Employment, ex parte EOC [1994] 1 All ER 910

$\mathrm{R} v$. Secretary of State for Transport, ex parte Factortame Ltd [1989] 2 CMLR 353; [1989] 2 All ER 692

$\mathrm{R} v$. Secretary of State for Transport, ex parte Factortame Ltd [1990] 2 AC 85; [1989] 3 CMLR 1

$\mathrm{R} v$. Secretary of State for Transport, ex parte Factortame Ltd award of interim relief: [1991] 1 All ER 70; [1990] 3 CMLR 375

SA Magnavision NV v. General Optical Council [1987] 1 CMLR 887; [1987] 2 CMLR 262 (Div. Ct.)

Webb v. EMO Cargo Ltd [1992] 2 All ER 43 (CA); [1993] 1 WLR 49 (HL); [1995] 4 All ER 577 (HL)

\section{German Courts}

Brunner [1994] 1 CMLR 57

$50,64,66,299$

Internationale Handelsgesellschaft [1974] 2 CMLR 549

189

Kloppenburg $v$. Finanzamt Leer [1989] 1 CMLR 873

295

Wunsche Handelsgesellschaft [1987] 3 CMLR 225

\section{Irish Courts}

Campus Oil $v$. Ministry for Industry and Energy [1984] 1 CMLR 479

\section{Belgian Courts}

Fromagerie Le Ski [1972] CMLR 330

\section{French Courts}

Boisdet [1991] 1 CMLR 3

Café Jacques Vabre [1975] 2 CMLR 336

260,295

Minister of the Interior v. Cohn-Bendit [1980] 1 CMLR 543

\section{D: Cases before the European Court of Human Rights}

Open Door and Dublin Well Woman $v$. Ireland (1992) Series A, no 246; (1993) 15 EHRR 244 


\section{Table of Treaties Establishing the European Communities and the European Union}

\begin{tabular}{|c|c|c|c|}
\hline \multicolumn{2}{|l|}{ EC } & \multicolumn{2}{|l|}{40} \\
\hline 2 & $9-10,77,89,374$ & 43 & $115,146,160,168,187,2$ \\
\hline (2) & 148 & 48 & $73-4,192,264,2$ \\
\hline & $9-10,77,374$ & $48(3)$ & \\
\hline A & $9,52,77$ & 50 & \\
\hline \multirow[t]{2}{*}{ B } & $50,53,78-80,84-5,96$ & 51 & \\
\hline & $102,141,143,198$ & 52 & $73,88,264,280-1$, \\
\hline $3 \mathrm{~B}(2)$ & 84 & 56 & \\
\hline & $80,107-8,141,172$ & 59 & $73,192-4,223,264$ \\
\hline A & 52,133 & 61 & \\
\hline \multirow[t]{3}{*}{1} & $6-18,76,116,207-9,212-13$ & 66 & 153,1 \\
\hline & $217,226-7,246,251,257$ & 71 & \\
\hline & $272-4,278,282,287,292$ & $73 \mathrm{~A}-7$ & $3 \mathrm{C}$ \\
\hline & $75,150,169,187,191$ & $73 C(2)$ & \\
\hline & 318 & 74 & \\
\hline 2) & $43,80,168-70,176$ & 75 & 150 \\
\hline 7) & 34 & 4 & \\
\hline A & $10,43,54,73,100$ & 85 & $1,86,115$ \\
\hline C & 114 & & $223,264,281,318,337,3$ \\
\hline 8 & 73 & $85(1)$ & \\
\hline 1) & 73 & 86 & $71,86,115,117,201,21$ \\
\hline 2) & 73 & & $223,264,281,318,3$ \\
\hline $3 \mathrm{~A}$ & $73-4,128$ & 90 & \\
\hline A(1) & 146 & $90(3)$ & $115,212,219,223,3$ \\
\hline (2) & 146,155 & 92 & 218 \\
\hline $3(1)$ & 73 & 93 & $117,218,2$ \\
\hline$B(2)$ & 73 & 93(2) & $222-3,33$ \\
\hline $8 \mathrm{C}$ & 73 & 93(3) & \\
\hline 8D & 129 & 95 & 245 \\
\hline $8 \mathrm{E}$ & $53,74,76$ & 95(2) & \\
\hline $73 \mathrm{H}$ & 100 & 97 & \\
\hline & & 100 & $80,146,157,168,1$ \\
\hline 30 & $264,280-1,293,298$ & $100-10$ & \\
\hline
\end{tabular}


Table of Treaties $\mathrm{xli}$

\begin{tabular}{|c|c|c|c|}
\hline $100 \mathrm{~A}$ & $\begin{array}{r}80,96,130,148,168 \\
175-7,219,227\end{array}$ & $\begin{array}{l}130 \mathrm{M} \\
130 \mathrm{R}(4)\end{array}$ & $\begin{array}{l}82 \\
82\end{array}$ \\
\hline $100 \mathrm{~A}(2)$ & 169 & $130 \mathrm{R}-\mathrm{T}$ & 131,171 \\
\hline $100 \mathrm{~A}(3)$ & 114,169 & $130 \mathrm{~S}$ & $150,175-6$ \\
\hline $100 \mathrm{~A}(4)$ & $96,117,218-19,309$ & $130 \mathrm{~S}(1)$ & 150 \\
\hline $100 \mathrm{~B}$ & 218 & $130 \mathrm{~S}(2)$ & 146 \\
\hline $100 \mathrm{C}$ & 124,145 & $130 \mathrm{~S}(3)$ & 153 \\
\hline $100 C(1)$ & 145 & $130 \mathrm{U}(2)$ & 196 \\
\hline $100 C(6)$ & 51 & $130 \mathrm{~W}$ & 150 \\
\hline $100 \mathrm{D}$ & 124 & 138 & 128 \\
\hline $102 \mathrm{~A}-109 \mathrm{M}$ & 52,220 & $138(2)$ & 125 \\
\hline 103 & 150 & $138(3)$ & 155,348 \\
\hline $104 \mathrm{~A}$ & 150 & $138 \mathrm{~A}$ & 127 \\
\hline 104B & 150 & 138B & 128 \\
\hline $105(6)$ & 128,155 & $138 \mathrm{C}$ & 129,173 \\
\hline $105 \mathrm{~A}$ & 133 & $138 \mathrm{D}$ & 129 \\
\hline $105 \mathrm{~A}(2)$ & 150 & $138 \mathrm{E}$ & 129 \\
\hline 106 & 133 & 140 & 126,128 \\
\hline $106(5)$ & 128,155 & 142 & 127 \\
\hline $109 \mathrm{C}(1)$ & 133 & 143 & 126 \\
\hline $109 \mathrm{C}(2)$ & 133 & 145 & $116,118,160-2$ \\
\hline $109 \mathrm{~F}$ & 133 & 146 & 117 \\
\hline 113 & 146 & 148 & 120 \\
\hline $113(1)$ & 80 & $148(2)$ & 120 \\
\hline 115 & $117,201,309,334$ & 151 & 123 \\
\hline 118 & $81,115,201$ & 152 & 118,128 \\
\hline $118(\mathrm{~A})$ & 150 & 155 & $114,117,160,210$ \\
\hline 119 & $89,184,246,296,373$ & $157(1)$ & 110 \\
\hline 125 & 150 & $157(2)$ & 110 \\
\hline 126 & 82,145 & 158 & $109-10$ \\
\hline $126(4)$ & 153 & $158(3)$ & 110 \\
\hline 127 & $79,82,150$ & 160 & 110 \\
\hline 128 & $75,121,131,151,153$ & 164 & $8,16,86,95,135$ \\
\hline $128(5)$ & 153 & & $181,251,303,316$ \\
\hline 129 & 150 & 167 & 134 \\
\hline $129(4)$ & 153 & $168 \mathrm{~A}$ & $43,133-4$ \\
\hline 129A & 373 & $168 \mathrm{~A}(3)$ & 134 \\
\hline $129 \mathrm{~A}(2)$ & 153 & 169 & $17,76,117,200,208$, \\
\hline 129D & 150,153 & & $210-12,214-16,218-21$, \\
\hline $129 \mathrm{D}(3)$ & 169 & & $223-4,226,228,243,263$, \\
\hline 130 & 151 & & $271,307,334,339$ \\
\hline $130 \mathrm{~A}$ & 335 & $169-171$ & $18,208,210-11$ \\
\hline $130 \mathrm{~A}-\mathrm{E}$ & 131 & $169-186$ & 135 \\
\hline $130 \mathrm{~A}-130 \mathrm{~T}$ & 43 & 170 & $17,208,210-11,215,218$, \\
\hline 130D & 128,155 & & 226,228 \\
\hline $130 \mathrm{E}$ & 150 & 171 & $14,135,210-12,221,226$, \\
\hline $130 \mathrm{I}$ & 153 & & 271,346 \\
\hline
\end{tabular}



329-31, 333-41, 343-9,

$$
\text { 354-6, } 364
$$

215(2)

173(1)

173(4)

174

174(2)

175

$305-6,310-12,314,320,343-9$

176 $306,337,354$

$17718,76,87,134,136,184,226$, 231-51, 254, 304, 307-8 $310-11,314-15,321,327$ $333,336,340-1,355-6$

177(2)

177(3)

178

180 (d)

184

186

188

$188 \mathrm{~A}-\mathrm{C}$

$188 \mathrm{~B}(7)$

189

$189 \mathrm{~A}$

189B

$189 \mathrm{~B}(8)$

$189 \mathrm{C}$

$189 \mathrm{C}(\mathrm{b})(1)$

190

191

193

194

198

198A-C

$198 \mathrm{C}$

198D-E

201

203

203(4)

203-4

209
239-41 235

341-3

$307,350,355,364$

220

238

Title VI

ECSC

$220,244,307$

351

132

132

4

18

31

273-4, 316, $327 \quad 35$

$52,150-3 \quad 65$

$156 \quad 88$

$43,148-50$

148

$142,183,335$

142

130

$130 \quad 54(2)$

$130 \quad 56(2)$

$130 \quad 57$

$131 \quad 57(1)$

$132 \quad 57(2)$

$164,166,177 \quad 100 \mathrm{~A}$

$166100 \mathrm{~B}$

$346 \quad 113$

$128 \quad 118 \mathrm{~A}$

$318 \quad 128$
135, 279, 281, 294, 299, 303, $306-7,310,312,314,316$,

354-7, 363-5

181,350

144

187

329

218

218

91

93

$91,128,155$

93

93, 196

90

$80-2,146,157,166,168$, 170-1, 176-7, 196-7, 294

90, 99

133

31

130

135

344

282

212

187,264

148,153

148,153

148,153

148

153

153

197, 153

153

147

148,150

$82,145,234$ 
Table of Treaties xliii

$\begin{array}{lrlr}\text { 130R(4) } & 96 & \text { J.3 } & 51,155 \\ \text { 130S } & 146 & \text { J.3(4) } \\ \text { 130S(3) } & 153 & \text { J.4 } & 51 \\ 149(1) & 144 & \text { J.4(2) } \\ 235 & 80-2,146,157,166,168, & \text { J.4(6) } & 51 \\ & 170-1,176-7,196-7,294 & \text { J.5 } & 32 \\ 238 & 90,99 & \text { J.5(3) } & 32 \\ & & \text { J.7 } & 156 \\ \text { SEA } & & \text { J.8 } & 125 \\ & & \text { J.8(1) } & 129,156 \\ 2 & 122,126-7,148 & \text { J.8(4) } & 123 \\ 30(1) & 44 & \text { J.9 } & 51,156 \\ 30(3)(b) & 43 & \text { J.11(2) } & 156 \\ 30(4) & 129 & \text { K.2(1) } & 156 \\ & & \text { K.3 } & 165 \\ \text { TEU } & & \text { K.3(2) } & 196 \\ & & \text { K.3(2)(c) } & 132 \\ \text { A } & 50,69,80 & \text { K.4 } & 51,156 \\ \text { A-F } & 136 & \text { K.4(2) } & 136,165,180 \\ \text { B } & 77-8,93,99 & \text { K.4(3) } & 51,123 \\ \text { C } & 99,108 & \text { K.6 } & 156 \\ \text { D } & 43,122 & \text { K.9 } & 156 \\ \text { E } & 108 & \text { L } & 123,156 \\ \text { F1 } & 69,194 & \text { N } & 51,145,156 \\ \text { F2 } & 50 & \text { Title VI } & 99 \\ \text { F(1) } & 93 & \text { Title V(CFSP) } \\ \text { F(2) } & 196 & \text { Title VI(CJHA) } & 57,66,258 \\ \text { J.1(2) } & & & 128,155 \\ & 56,74,88,188 & \text { O } & 121 \\ & & & 136 \\ & & & 136\end{array}$


xliv

\section{Table of UK Statutes}

British Telecommunications Act 1981

266

s. 23

Equal Pay Act 1970

296

s.1(2)(c)

European Communities Act 1972

ss. 2 and 3

$295,297,300$

s.2(1)

258

s.2(4)

296

s. 3

296-7

204

Merchant Shipping Act 1988

19, 221, 293, 296

Sex Discrimination Act 1975

297, 298 


\section{List of Abbreviations}

\begin{tabular}{ll} 
AC & Appeal Cases \\
AJCL & American Journal of Comparative Law \\
AJIL & American Journal of International Law \\
All ER & All England Law Reports \\
BEUC & Bureau Européen des Unions Consommateurs \\
CAP & Common Agricultural Policy \\
CCT & Common Customs Tariff \\
CE & Compulsory expenditure \\
CEDEFOP & European Centre for the Development of Vocational \\
& Training \\
CEEP & Comite Européen des Entreprises Publiques \\
CFSP & Common Foreign and Security Policy \\
CJHA & Cooperation in Justice and Home Affairs \\
CMLR & Common Market Law Reports \\
CMLRev. & Common Market Law Review \\
Col. LRev. & Columbia Law Review \\
Comp. Pol. Studs. & Comparative Political Studies \\
COPA & Confederation of Professional Agricultural \\
& Organizations \\
COREPER & Committee of Permanent Representatives \\
CREW & Centre for Research on European Women \\
DG & Directorate General \\
DTEU & Draft Treaty establishing a European Union \\
EBLR & European Business Law Review \\
EC & European Community \\
ECB & European Central Bank \\
ECHR & European Convention on Human Rights \\
ECLR & European Competition Law Review \\
ECOSOC & Economic and Social Committee \\
ECR & European Court Reports \\
ECSC & European Coal and Steel Community \\
EDC & European Defence Community \\
EEA & European Economic Area \\
EEC & European Economic Community \\
EEIG & European Economic Interest Grouping \\
EFTA & European Free Trade Association \\
EIB & European Investment Bank \\
EJIL & European Journal of International Law \\
& \\
\hline &
\end{tabular}




\begin{tabular}{|c|c|}
\hline$E L J$ & European Law Journal \\
\hline ELRev. & European Law Review \\
\hline EMI & European Monetary Institute \\
\hline EMS & European Monetary System \\
\hline EMU & Economic and Monetary Union \\
\hline EPC & European Political Corporation \\
\hline$E P L$ & European Public Law \\
\hline ERM & Exchange Rate Mechanism \\
\hline$E R P L$ & European Review of Private Law \\
\hline ERTA & European Road Transport Agreement \\
\hline ESCB & European System of Central Banks \\
\hline ETUC & European Trade Union Confederation \\
\hline EU & European Union \\
\hline EUI & European University Institute \\
\hline Euratom & European Atomic Energy Community \\
\hline Eur. J. Pol. Res. & European Journal of Political Research \\
\hline Eur. Pub. $L$ & European Public Law \\
\hline FAO & Food and Agriculture Organization (of UN) \\
\hline$F S R$ & Fleet Street Reports \\
\hline FYROM & Yugoslav Republic of Macedonia \\
\hline GATT & General Agreement on Tariffs and Trade \\
\hline GNP & Gross National Product \\
\hline$I C L Q$ & International and Comparative Law Quarterly \\
\hline IGC & Intergovernmental Conference \\
\hline$I L J$ & Industrial Law Journal \\
\hline Intl. Aff. & International Affairs \\
\hline ILO & International Labour Organization \\
\hline IO & International Organization \\
\hline$I R L R$ & Industrial Relations Law Reports \\
\hline$J B L$ & Journal of Business Law \\
\hline$J C M S$ & Journal of Common Market Studies \\
\hline$J E P P$ & Journal of European Public Policy \\
\hline$J S W F L$ & Journal of Social Welfare and Family Law \\
\hline LIEI & Legal Issues of European Integration \\
\hline$L Q R$ & Law Quarterly Review \\
\hline$L S$ & Legal Studies \\
\hline MCAs & Monetary Compensatory Amounts \\
\hline MEP & Member of the European Parliament \\
\hline$M J$ & Maastricht Journal of European and Comparative Lan \\
\hline$M L R$ & Modern Law Review \\
\hline NCE & Non-compulsory expenditure \\
\hline$N I L Q$ & Northern Ireland Legal Quarterly \\
\hline OECD & $\begin{array}{l}\text { Organization for Economic Cooperation and } \\
\text { Development }\end{array}$ \\
\hline OEEC & Organization for European Economic Cooperation \\
\hline$O J$ & Official Journal \\
\hline OJLS & Oxford Journal of Legal Studies \\
\hline
\end{tabular}


OOPEC

$P L$

QMV

SEA

TEU

UN

UNICE

VAT

WEU

W. Eur. Pols.

WTO

$Y E L$
Office of Official Publications of the European Communities

Public Law

Qualified Majority Voting

Single European Act

Treaty on European Union

United Nations

Union of Industries of the European Community

Value Added Tax

West European Union

West European Politics

World Trade Organisation

Yearbook of European Law 